\title{
ADAPTABILIDAD DE HÍBRIDOS PRECOMERCIALES TROPICALES DE MAÍZ EN EL BAJÍO DE GUANAJUATO, MÉXICO
}

\author{
ADAPTABILITY OF TROPICAL PRE-COMERCIALS HYBRIDS \\ OF CORN IN THE BAJIO OF GUANAJUATO, MEXICO
}

\section{J. Guadalupe García Rodríguez ${ }^{1}$, Mariano Mendoza-Elos ${ }^{2}$, Francisco Cervantes Ortiz $^{3}$, Juan Gabriel Ramírez-Pimentel ${ }^{4}$, Cesar Leobardo Aguirre-Mancilla ${ }^{5}$, Marco Antonio García-Perea ', María Guadalupe Figueroa Rivera?, Gilberto Rodríguez Pérez ${ }^{8}$, Sergio A. Rodríguez-Herrera ${ }^{9}$}

\footnotetext{
${ }^{1}$ Maestro en ciencias en Producción y Tecnología de semillas, ${ }^{2}$ Doctor en Ciencias en Fitomejoramiento, ${ }^{3}$ Doctor en Ciencias en Producción de Semillas, ${ }^{4}$ Doctor en Ciencias en Alimentos, ${ }^{5}$ Doctor en Ciencias en Biotecnología de Plantas,

${ }^{6}$ Doctor en Ciencias en Genética, ${ }^{7}$ Doctora en Ciencias en Materiales, ${ }^{8}$ Doctor en Ciencias Agrícolas, ${ }^{9}$ Doctor en Ciencias Agrarias, Universidad Autónoma Agraria Antonio Narro.

1,2,3,4,5,7,8 Instituto Tecnológico de Roque. México, ' ${ }^{7}$ Facultad de Estudios Superiores Cuautitlán, UNAM, México, ${ }^{9}$ Universidad Autónoma Agraria Antonio Narro. Coahuila

Autor de correspondencia: mmendoza66@hotmail.com
}

\section{RESUMEN}

El objetivo de este estudio fue identificar la mejor fecha de siembra de nuevas variedades experimentales de maíz y seleccionar los mejores genotipos. El trabajo se realizó en el campo experimental del Instituto Tecnológico de Roque, Celaya, Gto. El material genético de maíz fue un ensayo de variedades procedentes del Centro Internacional de Mejoramiento de Maíz y Trigo (CIMMYT), evaluado durante dos fechas de siembra: 25 de junio de 2016 (temporal) y 25 de marzo de 2017 (riego). Se midieron las variables: días a la floración masculina y femenina, altura de planta, altura de mazorca y rendimiento. Estas fueron analizadas con base en el modelo del diseño Látice con tres repeticiones; la comparación de medias se realizó mediante la prueba de diferencia mínima significativa (DMS). Los resultados indican diferencias estadísticas altamente significativas para tratamientos y fechas de siembra. La mejor fecha de siembra fue el 25 de marzo al favorecer una producción de $12.4 \mathrm{t} \mathrm{ha}^{-1}(12 \%)$, más que la fecha de siembra 25 de junio, que alcanzó 11.1 t ha-1. En rendimiento de grano, los genotipos 9 (RS98TLW-5 B) y 6 (RS98TLW-2 B) fueron los mejores presentando rendimientos de $13.0 \mathrm{y}$ $14.0 \mathrm{t} \mathrm{ha}^{-1}$, respectivamente.

Palabras clave: Adaptabilidad, fechas de siembra, variedades 


\section{SUMMARY}

The objective of the present study was to identify the best sowing date for new experimental varieties of corn and also, to select the best corn genotypes. The work was carried out in the experimental field of the Instituto Tecnologico de Roque, Celaya, Gto., Mexico. The corn genetic material was provided by CIMMYT and evaluated for two sowing dates, June $25^{\text {th }}$ (rainfed) and March 25 $5^{\text {th }}$ (irrigation) of 2016 and 2017, respectively. The following variables were measured: days at male and female flowering, plant height, cob height and yield. The study was conducted as a Latice experimental design with three replicates. Mean comparison was performed by Least Significant Difference (LSD). Statistically significant differences for treatments and sowing dates were detected. The best sowing date was March 27th, favoring a corn grain production of $12.4 \mathrm{t} \mathrm{ha}^{-1}$ (12\%, moisture content basis), compared to the $11.1 \mathrm{t} \mathrm{ha}^{-1}$ yield for June 25th sowing date. In grain yield, the genotypes 9 (RS98TLW-5 B) and 6 (RS98TLW-2 B) were the best with recoveries of 13.0 and $14.0 \mathrm{t} \mathrm{ha}^{-1}$, respectively.

Key words: Adaptability, dates of sowing, varieties

\section{Resumen gráfico}

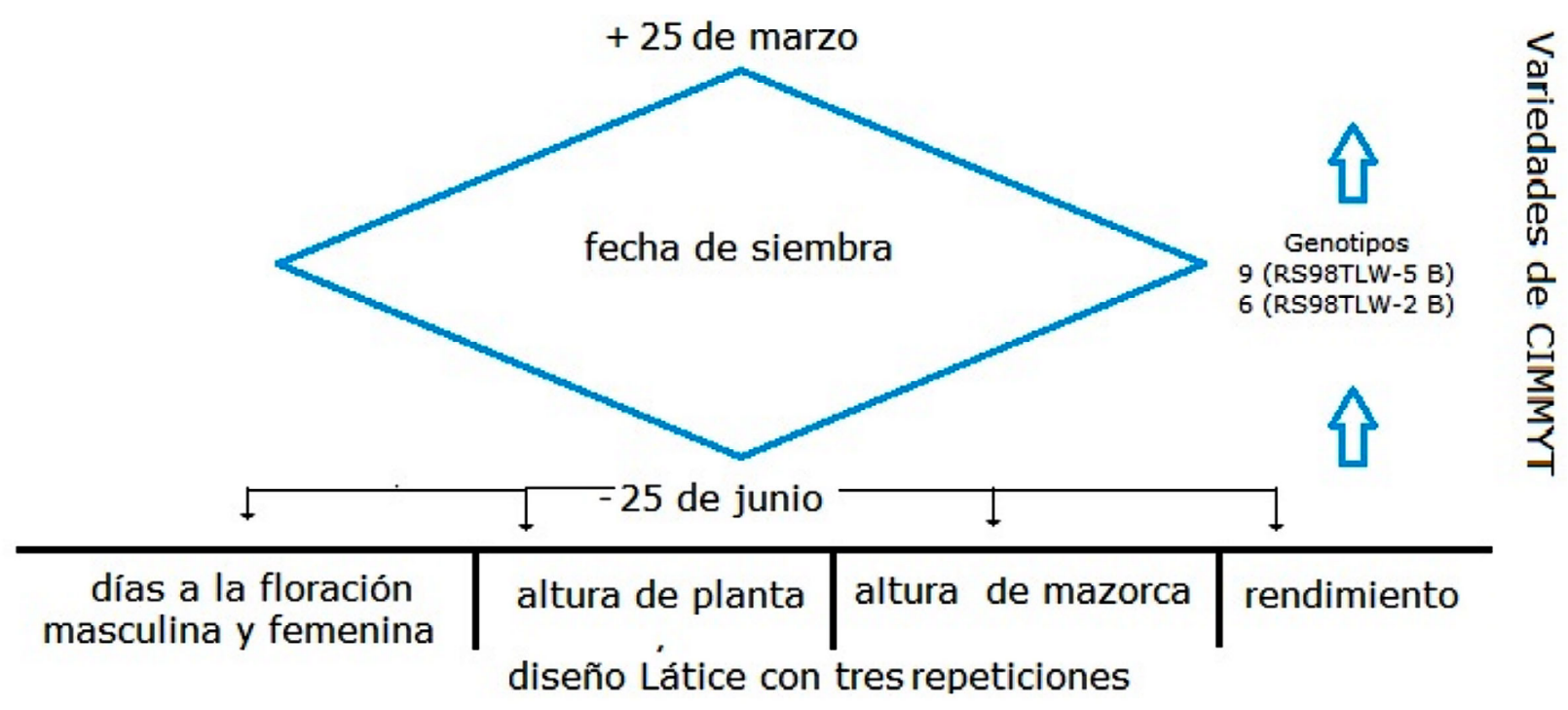

\section{INTRODUCCIÓN}

A nivel mundial y nacional, el cultivo de maíz ocupa el primer lugar en superficie sembrada. El Departamento de Agricultura de Estados Unidos (USDA, por sus siglas en inglés) en junio de 2015 estimó una producción mundial de 989.3 millones de toneladas. México se encuentra en el séptimo lugar con una producción de $2.3 \%$ (USDA, 2015). El estado de
Guanajuato cuenta con una gran superficie para la producción de grano de maíz; sin embargo, el estado es considerado una región semiárida con graves problemas de agua; cabe mencionar que de las 385,743 has que se han sembrado con anterioridad, $69.89 \%$ son de temporal y $30.11 \%$ son de riego (SIAP, 2017). González (1995) menciona que, del total de 
maíz utilizado en el país, 59 \% se consume en tortillas, $36 \%$ se destina a otros usos (animal y semillas) y $5 \%$ es procesado por la industria almidonera. El consumo per cápita es de $300 \mathrm{~g} \mathrm{día}^{-1}$, con lo cual se aporta el $56 \%$ de las calorías y el $47 \%$ de las proteínas de la alimentación del mexicano. En áreas rurales, estos porcentajes son $70 \%$ y $56 \%$, respectivamente.

Las características y comportamiento que debe reunir una nueva variedad mejorada son las que determinan su identidad y uniformidad; cuyos rasgos morfológicos, fisiológicos o químicos permiten diferenciarlas de otras variedades, a pesar de la similitud debida a las pequeñas diferencias genéticas (Copeland, 1976). Además, la Asociación de Analistas Oficiales de Semillas (AOSA) señalan en su definición de variedad "que los caracteres varietales deben cumplir con tres funciones específicas: diferente, en el sentido de que la variedad se puede identificar por una o más características morfológicas, físicas o de otro tipo que las distinguen de las otras variedades conocidas; uniforme, en el sentido que se pueden describir la variación de las características esenciales y típicas; y estable, por cuanto la variedad permanecerá sin cambios y tendrá un grado razonable de confiabilidad en sus características esenciales y típicas, y en su uniformidad al producirla o reconstruirla según lo exigen las diferentes categorías de la variedad".

Molina (1992) indica que la inconsistencia del comportamiento de las variedades a través de ambientes de evaluación, puede tener su origen en el grado de diversidad de los genotipos y de los ambientes de prueba, también señala, que tal inestabilidad es producto de la interacción genotipo-ambiente no lineal, por lo que la dirección y magnitud resultantes, en general, es impredecible. Por su parte, Palomo (1976) menciona que al evaluar diferentes variedades en un ambiente, las diferencias observadas entre las variedades se confunden con los efectos de la interacción genotipo-ambiente; y para recomendar una variedad en una región, es necesario evaluar varias variedades en una muestra adecuada de ambientes.

Por lo anterior, los objetivos de esta investigación fueron: 1) identificar la mejor fecha de siembra en variedades de maíz y 2) seleccionar los mejores genotipos de maíz.

\section{MATERIALES Y MÉTODOS}

El experimento se realizó en el Instituto Tecnológico de Roque, Celaya, Gto., durante 2016 y 2017, ubicado en el km 8 carretera CelayaJuventino Rosas, cuyas coordenadas son: $20^{\circ}$ $34^{\prime} 00^{\prime \prime}$ Latitud norte y $100^{\circ} 50^{\prime}$ 00" Longitud oeste, a una altura de 1765 msnm.

El clima de la región de Roque es BS1 hW (e) semicálido, clasificación de Köppen, modificada por García (1973), una altitud de 1767 msnm, considerado como subtropical (INEGI, 2012). La temperatura media anual es de 18 ${ }^{\circ} \mathrm{C}$, las extremas varían de $-0.3{ }^{\circ} \mathrm{C}$ hasta 35 ${ }^{\circ} \mathrm{C}$. La precipitación media anual oscila entre 400 y 700 mm; los suelos son de origen volcánico, ricos en potasio, calcio y magnesio, con textura arcillosa, pobres en materia orgánica, $\mathrm{pH}$ de 8.0 (medianamente alcalino), punto de marchitez permanente de $21 \%$ y densidad aparente de $1.2 \mathrm{~g} \mathrm{~cm}^{3}$ (SARH-INIFAP-PROCATI, 1991).

El material genético de maíz utilizado fue un ensayo de variedades del CIMMYT, evaluado en dos fechas de siembra: 25 de junio de 2016 (temporal) y 25 de marzo de 2017 (riego). Los materiales fueron: variedades de tierras tropicales de madurez tardía y grano blanco: 1. RP73NIC1, 2. RP73NIC2, 3. RP76NIC1, 4. 
RP76SAL1, 5. RS98TLW-1 B, 6. RS98TLW-2 B, 7. RS98TLW-3 B, 8. RS98TLW-4 B, 9. RS98TLW-5 B, 10. RS98TLW- $1 A B, 11$. RS98TLW-2 $A B, 12$. RS98TLW-3 AB, 13. RS99TLW BNSEQ (1), 14. RS99TLW BNSEQ (2), 15. RS99 TLWQ, 16. RS99 TLWQ-1, 17. RS97 TLW GH "A ", 18. RS97 TLW GH "A y B" (2), además se integraron como testigo 19. P1C2 (T) y 20. ANTILOPE (T).

La preparación del suelo en los dos ciclos se realizó 20 días previos a la siembra. Consistió en un barbecho de $30 \mathrm{~cm}$ de profundidad y dos pasos de rastra. Posteriormente, se trazaron los surcos a una distancia de $0.75 \mathrm{~m}$. El tamaño de la unidad experimental fue un surco de $5 \mathrm{~m}$ de longitud, con una separación de $0.75 \mathrm{~m}$ y entre plantas de $0.20 \mathrm{~m}$. La parcela útil fue de $3.9 \mathrm{~m}^{2}$.

La siembra de los genotipos se realizó el 25 de junio del 2016 (temporal) y el 25 de marzo del 2017 (riego). Se llevó a cabo en forma manual, depositando dos semillas por golpe cada $20 \mathrm{~cm}$. El aclareo de plantas se efectuó 45 días después de la siembra, para tener una densidad de 66500 plantas ha-1.

La fertilización en las dos fechas de siembra fue con la fórmula 240-80-00. Se aplicó la mitad de nitrógeno y todo el fósforo al momento de la siembra; 40 días después se aplicó la otra mitad de nitrógeno. Como fuente de nitrógeno se utilizó urea (46\% N), para fósforo, superfosfato de calcio triple $\left(46 \% \mathrm{P}_{2} \mathrm{O}_{5}\right)$.

Las variables evaluadas por unidad experimental fueron: días a floración masculina y femenina (DFM, DFF). Consideradas, como el número de días transcurridos desde el día de la siembra hasta observar el $50+1 \%$ de las plantas con espigas dehiscentes y estigmas expuestos, respectivamente. Altura de planta y mazorca ( $A P, A M Z$ ). En la etapa de madurez fisiológica se midió la longitud de tres plantas desde la base de la planta hasta la base de la espiga y el punto de inserción de la mazorca, respectivamente. El rendimiento (REND) en $t$ $\mathrm{ha}^{-1}$ fue estimado en grano y ajustado al $14 \%$ de humedad.

Las variables fueron analizadas con base en el modelo del diseño de Látice con bloques incompletos. La comparación de medias se realizó bajo la prueba de diferencia mínima significativa (DMS) con el paquete SAS versión 6.12 .

\section{RESULTADOS Y DISCUSIÓN}

En la Tabla 1 se presentan los cuadrados medios del análisis de varianza (ANOVA) de las variables bajo estudio para variedades tropicales de maíz y grano blanco. En la fuente de variación fechas de siembra hubo diferencias significativas al 1 por ciento de probabilidad para todas las características, con un coeficiente de variación de 2.6, 2.7, 11.3, 17.7 y $15.4 \%$ para DFM, DFF, AP, AMZ y REND, respectivamente. En la fuente Tratamientos (variedades) también se detectaron diferencias al uno por ciento de probabilidad, lo cual indica que existe variabilidad genética entre los materiales, debido a su origen; esto permite al fitomejorador realizar una selección efectiva de los genotipos. En la interacción fecha de siembra $x$ tratamiento no se encontraron diferencias estadísticas, lo que se atribuyen a la genética de los materiales bajo estudio, al manejo agronómico, ambiente y conducción del experimento (Mendoza et al., 2006; Wong et al., 2007; Cervantes, 2016). 
Tabla 1. Cuadrados medios del ANOVA combinado en variedades de maíz tropicales y grano blanco en el 2016 y 2017 en la localidad de Celaya, Guanajuato, México.

\begin{tabular}{|c|c|c|c|c|c|c|}
\hline Fuente de Variación & GI & $\begin{array}{c}\text { DFM } \\
\text { (días) }\end{array}$ & $\begin{array}{c}\text { DFF } \\
\text { (días) }\end{array}$ & $\begin{array}{l}\text { AP } \\
(\mathrm{m})\end{array}$ & $\begin{array}{c}A M Z \\
(m)\end{array}$ & $\begin{array}{c}\text { REND } \\
\left(t h a^{-1}\right)\end{array}$ \\
\hline Fechas & 1 & $4800.7 * *$ & $5386.8 * *$ & $0.99 * *$ & $0.75 * *$ & $52.1 * *$ \\
\hline Bloque & 6 & 7.5 & 6.62 & 0.01 & 0.01 & 2.3 \\
\hline Tratamiento & 19 & $16.9 * *$ & $18.3 * *$ & $0.11 * *$ & $0.14 * *$ & $12.3 * *$ \\
\hline Fecha $x$ Tratamiento & 19 & $5.8 \mathrm{NS}$ & $5.3 \mathrm{NS}$ & $0.03 \mathrm{NS}$ & $0.03 *$ & $4.9 \mathrm{NS}$ \\
\hline Error & 74 & 3.7 & 4.0 & 0.04 & 0.02 & 3.3 \\
\hline Total & 116 & ---- & ---- & ---- & ---- & ---- \\
\hline C. V. (\%) & ---- & 2.6 & 2.7 & 11.3 & 17.7 & 15.4 \\
\hline
\end{tabular}

*,**, Significativo al 0.05 y 0.01 , respectivamente. GI = Grados de libertad; AP = Altura de la planta. NS = No significativo. $A M Z$ = Altura de la mazorca. DFM = Días a la floración masculina.

DFF = Días a la floración femenina REND = Rendimiento.

Para la fecha de siembra establecida el 25 de marzo (riego), los genotipos presentaron una respuesta de ciclo vegetativo tardío con 80 y 82 días a floración masculina y femenina, respectivamente. De igual manera, el promedio para altura de planta también se incrementó en forma estadística. En el caso del rendimiento, esta fecha (riego) superó en $1.3 \mathrm{t} \mathrm{ha}^{-1}$ a la siembra realizada el 25 de junio, en esta última fecha influyeron las condiciones de precipitación, fotoperíodo y humedad, afectando a todas las variables bajo estudio (Tabla 2). Al respecto, Reyes (1990) propone que la mejor fecha de siembra para variedades tardías e intermedias en el Bajío corresponde del 15 de marzo al 30 de mayo. $Y$ para este mismo ambiente, las fechas de siembra propuestas para temporal son en el mes de junio y julio. Se puede observar en esta misma tabla, que el ciclo a madurez traducido con días a floración y alturas de planta y mazorca se acortan en esta fecha, resultados que coinciden con Alejo et al. (2006) y Almaraz (2015).

Tabla 2. Prueba de medias de las características evaluadas a través de dos años en variedades tropicales y grano blanco de maíz en Celaya, Guanajuato, México.

\begin{tabular}{|c|c|c|c|c|c|}
\hline Fechas de siembra & $\begin{array}{c}\text { DFM } \\
\text { (días) }\end{array}$ & $\begin{array}{c}\text { DFF } \\
\text { (días) }\end{array}$ & $\begin{array}{l}\text { AP } \\
(\mathrm{m})\end{array}$ & $\begin{array}{c}\text { AMZ } \\
(\mathrm{m})\end{array}$ & $\begin{array}{l}\text { REND } \\
\left(\text { t ha }^{-1}\right)\end{array}$ \\
\hline 25/marzo/2017 (riego) & $80 \mathrm{a}$ & $82 \mathrm{a}$ & $1.76 \mathrm{a}$ & $0.80 \mathrm{a}$ & $12.4 \mathrm{a}$ \\
\hline 25/junio/2016 (temporal) & $68 \mathrm{~b}$ & $68 \mathrm{~b}$ & $1.57 b$ & $0.64 b$ & $11.1 \mathrm{~b}$ \\
\hline DMS (5\%) & 0.7 & 0.7 & 0.05 & 0.03 & 0.4 \\
\hline
\end{tabular}

Letras iguales indican que no hay diferencia estadística entre fechas. DFM = Días a la floración masculina. DFF = Días a la floración femenina. $A P=$ Altura de planta. AMZ = Altura de mazorca. REND = Rendimiento.

En la Tabla 3 se presenta la prueba DMS al a $=0.05$ para DFM, se observa que el testigo 19 presentó la emisión de polen en un período máximo de 78 días. En tanto que el testigo 20 lo emitió a los 70 días; la diferencia fue de ocho días. El genotipo 14 resultó estadísticamente igual al testigo 19, y representa el $5 \%$ del universo de los genotipos. El $100 \%$ resultaron de ciclo intermedio, 
al variar de 71 a 76 días con respecto al testigo 20 (70 días); la diferencia entre ellos oscila de uno a cinco días. Estos resultados coinciden con CIMMYT (2015) al evaluar ensayos de variedades subtropicales en Roque, Celaya, Gto. Al respecto, Reyes (1990) y CIMMYT (1999) indican que las variedades de maíz tardías emiten polen de 81 a 90 días, las intermedias de 71 a 80 y las precoces de 60 a 70 días.

Tabla 3. Medias para las variables agronómicas evaluadas en variedades de maíz tropicales en 2016 y 2017 en la localidad de Celaya, Gto., México.

\begin{tabular}{|c|c|c|c|c|c|}
\hline $\begin{array}{l}\text { TRATA- } \\
\text { MIENTO }\end{array}$ & $\begin{array}{c}\text { DFM } \\
\text { (días) }\end{array}$ & $\begin{array}{c}\text { DFF } \\
\text { (días) }\end{array}$ & $\begin{array}{l}\text { AP } \\
(m)\end{array}$ & $\begin{array}{c}\text { AMZ } \\
(\mathrm{m})\end{array}$ & $\begin{array}{l}\text { REND } \\
\left(\mathrm{t} \mathrm{ha} \mathrm{h}^{-1}\right)\end{array}$ \\
\hline 20 & 70 & 72 & 1.73 & 0.75 & 14.9 \\
\hline 6 & 73 & 73 & 1.67 & 0.67 & 14.0 \\
\hline 9 & 74 & 75 & 1.62 & 0.65 & 13.0 \\
\hline 19 & 78 & 79 & 2.16 & 1.33 & 12.7 \\
\hline 18 & 74 & 75 & 1.69 & 0.69 & 12.7 \\
\hline 11 & 74 & 75 & 1.65 & 0.65 & 12.5 \\
\hline 13 & 74 & 75 & 1.77 & 0.74 & 12.4 \\
\hline 16 & 74 & 76 & 1.45 & 0.60 & 12.0 \\
\hline 8 & 73 & 73 & 1.58 & 0.66 & 12.0 \\
\hline 14 & 76 & 75 & 1.65 & 0.80 & 11.8 \\
\hline 17 & 75 & 77 & 1.59 & 0.73 & 11.7 \\
\hline 5 & 75 & 75 & 1.62 & 0.70 & 11.5 \\
\hline 12 & 75 & 76 & 1.63 & 0.64 & 11.4 \\
\hline 7 & 71 & 71 & 1.61 & 0.70 & 11.1 \\
\hline 10 & 75 & 75 & 1.70 & 0.71 & 11.0 \\
\hline 15 & 75 & 75 & 1.60 & 0.60 & 11.0 \\
\hline 3 & 73 & 74 & 1.67 & 0.75 & 10.2 \\
\hline 2 & 74 & 76 & 1.66 & 0.73 & 9.9 \\
\hline 4 & 73 & 74 & 1.62 & 0.65 & 9.6 \\
\hline 1 & 74 & 75 & 1.59 & 0.65 & 9.0 \\
\hline Media & 74 & 75 & 1.66 & 0.72 & 11.7 \\
\hline DMS (5 \%) & 2.2 & 2.3 & 0.22 & 0.15 & 2.1 \\
\hline
\end{tabular}

DFM = Días a la floración masculina, $\mathrm{AMZ}=$ Altura de mazorca. DFF = Días a la floración femenina. REND = Rendimiento. AP = Altura de planta.
Para DFF se detectaron cinco grupos estadísticos. El testigo 19 presentó los estigmas receptivos en un máximo de 79 días, mientras que el tratamiento 7 los emitió a los 71 días. El genotipo 17 con 77 días, resultó estadísticamente igual al genotipo 19. Los genotipos 6,7 y 8 con un día de diferencia, resultaron estadísticamente igual al testigo 20 , y equivalen al $17 \%$ del total; en tanto que el $100 \%$ de ellos se comportaron de ciclo intermedio, con un rango de 71 a 79 días. Estas evidencias coinciden con Balderas (1991) y CIMMYT (2015). Al respecto, López (1991) indica que la velocidad y calidad del desarrollo floral deben estar en equilibrio para que exista una sincronización entre la emisión de polen y la aparición de los estigmas.

El testigo 19 alcanzó la altura máxima de 2.16 $\mathrm{m}$, fue $0.39 \mathrm{~m}$ más alto que el segundo grupo representado por el genotipo 13, con $1.77 \mathrm{~m}$. Mientras que el genotipo 16 presentó la menor altura de planta con $1.45 \mathrm{~m}$. El $94 \%$ de los genotipos en estudio igualaron estadísticamente al testigo 20 con $1.73 \mathrm{~m}$ y presentaron una media general de $1.66 \mathrm{~m}$.

En la comparación de medias (DMS $a=0.05$ ) para altura de mazorca, se definieron cuatro grupos estadísticos. El testigo 19 fue el tratamiento que presentó la altura de mazorca mayor con $1.33 \mathrm{~m}, 32 \%$ superior que el segundo grupo, representado por el genotipo 14, con $0.80 \mathrm{~m}$. Los genotipos 15 y 16 mostraron una altura de $0.60 \mathrm{~m}$, la diferencia fue de 25 a 55 $\%$, respectivamente. Los genotipos presentaron una media general de $0.72 \mathrm{~m}$.

En la Figura 1 se muestra la comparación de medias de la interacción fechas $x$ tratamientos, donde se observa que el genotipo 6 presentó en las dos fechas de siembra igual altura de mazorca $(0.67 \mathrm{~m})$; el genotipo 11 en la fecha de siembra de 25 de junio (temporal) presentó 
mayor altura. Con relación a la fecha de 25 de marzo (riego), las plantas presentaron menor altura de mazorca $(0.63 \mathrm{~m})$, con una diferencia entre ellos de $6 \%$. También se aprecia el mismo patrón de comportamiento en altura de mazorca, la cual es afectada por las condiciones climáticas y edáficas. Los genotipos $6,8,10,11$ y 12 presentaron una respuesta uniforme en las dos fechas de siembra, por lo tanto, el aspecto genético juega un papel importante en la plasticidad o respuesta al ambiente. Sin embargo, los genotipos 2, 3, 14 y el testigo 19 en la fecha de 25 de marzo, presentaron una respuesta mejor a las condiciones ambientales, debido a que alcanzaron mayor altura del punto de inserción de la mazorca, la cual es una desventaja cuando se realiza la cosecha mecánica. Con base en la floración, estos genotipos podrían clasificarse como de ciclo intermedio.

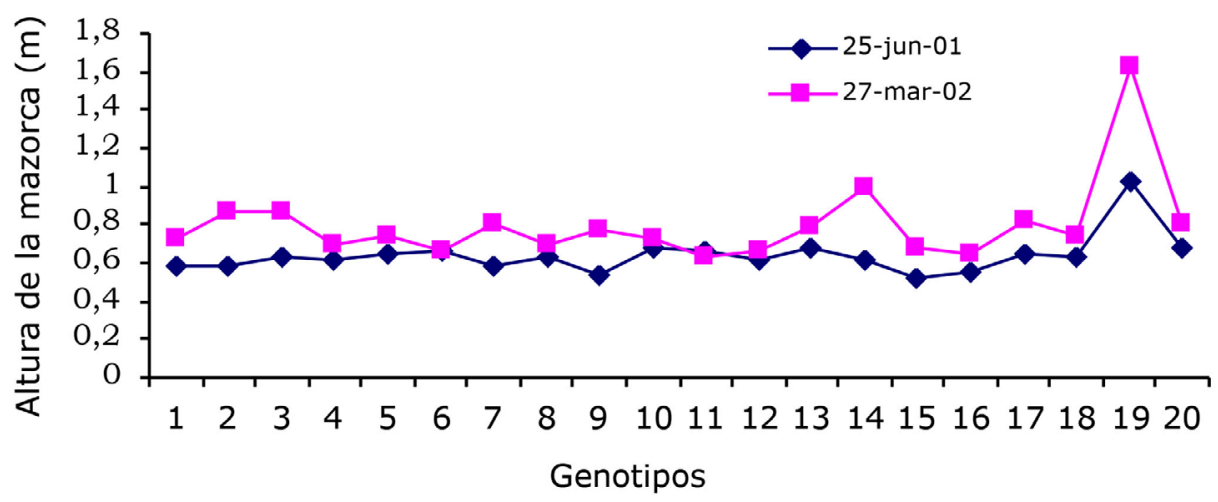

Figura 1. Comparación de medias de la interacción fechas x tratamientos para AMZ de genotipos tropicales de maíz en el 2016 y 2017 en Celaya, Gto., México.

En la variable REND en promedio de los dos años de evaluación, el testigo 20 presentó el rendimiento máximo (14.9 $\left.\mathrm{t} \mathrm{ha}^{-1}\right)$. Mientras que el genotipo 1 mostró el rendimiento mínimo (9.0 $\mathrm{t} \mathrm{ha}^{-1}$ ), la diferencia fue de $5.9 \mathrm{t} \mathrm{ha}$ 1. Los genotipos 9 y 6 , con rendimientos de 13.0 a $14.0 \mathrm{t} \mathrm{ha}^{-1}$, igualaron estadísticamente al testigo 20, y representan al $10 \%$ del total evaluado. Resultados estadísticamente similares se encontraron en las variables DFM y DFF, esto permite realizar la selección de los genotipos 9 y 6, como los sobresalientes a nivel experimental para estos caracteres. Los genotipos $14,8,16,13,11$ y 18 , presentaron un rendimiento aceptable con valores de 11.8 a $12.7 \mathrm{t} \mathrm{ha}^{-1}$.

Estos resultados demuestran que no existe una relación positiva, es decir, los genotipos con mayor altura no son los más productivos, en concordancia con Sinchi (1991). Por ejemplo, el genotipo 9 presentó una altura de planta de $1.62 \mathrm{~m}$ y rendimiento de $13.0 \mathrm{t} \mathrm{ha}^{-1}$, el 6 con $1.67 \mathrm{~m}$ de altura produjo $14.0 \mathrm{t} \mathrm{ha}^{-1} \mathrm{y}$ el testigo 20 con $1.73 \mathrm{~m}$ de altura arrojó un rendimiento de $14.9 \mathrm{t} \mathrm{ha}^{-1}$. Por otro lado, el genotipo 19 con una altura de 2.16 m presentó un rendimiento menor a $2.2 \mathrm{t} \mathrm{ha}^{-1}$, y el genotipo 13 con altura de $1.77 \mathrm{~m}$ presentó $1.6 \mathrm{t} \mathrm{ha}^{-1}$ menos que el genotipo 6 .

\section{CONCLUSIONES}

La mejor fecha de siembra para las variables evaluadas y para rendimiento de grano fue el 25 de marzo (riego) al favorecer una producción de $1.3 \mathrm{t} \mathrm{ha}^{-1}(12 \%)$, más que la fecha de siembra 25 de junio (temporal) que alcanzó $11.1 \mathrm{t} \mathrm{ha}^{-1}$. 
En rendimiento de grano, los genotipos 9 y 6 presentaron 13.0 y $14.0 \mathrm{t} \mathrm{ha}^{-1}$, respectivamente, estadísticamente igual al testigo (14.9 t ha$\left.{ }^{1}\right)$, y los genotipos 16 y 18 con 12.0 y 12.7 t ha1 , se ubicaron por arriba de la media general $\left(11.7 \mathrm{t} \mathrm{ha}^{-1}\right)$.

\section{LITERATURA CITADA}

Almaraz, V.C.A. (2017). Comportamiento agronómico y de calidad de semilla de maíces híbridos tropicales y subtropicales. Tesis de Maestría. Producción y Tecnología de Semillas. Instituto Tecnológico de Roque, Celaya, Gto., México. 71 p.

Alejos, G., Monasterio, P., y Rea, R. (2006). Análisis de la interacción genotipo ambiente para el rendimiento de maíz en la región maicera del estado de Yaracuy, Venezuela. Agron. Trop. 56(3):369-384.

Balderas, P, G. (1991). Caracterización de líneas y cruzas de maíz y su comportamiento agronómico en temporal. Tesis de Maestría. UG. Guadalajara, Jal. $140 \mathrm{pp}$.

Cervantes J. C. (2016). Aptitud combinatoria entre líneas de maíz de diferente grupo heterótico. Tesis de Maestría. Producción y Tecnología de Semillas. Instituto Tecnológico de Roque, Celaya, Gto., México. 70 p.

CIMMYT. (1999). Manejo de los ensayos e informe de los datos para el Programa de Ensayos Internacionales de Maíz. Quinta reimpresión. México. D. F. 20 p.

CIMMYT. (2015). Programa de Ensayos Internacionales de Maíz. Informe final de 1999. Ed. CIMMYT. México. 43-151.

Copeland, L.O. (1976). Principles of seed science and technology. Burguess Pub.Co. Minneapolis, Minnesota. 369 p.

García, E. (1973). Modificaciones al sistema de clasificación climática de Köppen. Ed. UNAM. Instituto de Geografía. México, D. F. 245 p.

González, A.V. (1995). El maíz y su conservación. Ed. Trillas. Primera Edición. México. 11-13.

INEGI, 2010. Topografía. Compendio de Información Geográfica Municipa. [En línea] Available at: http://www.inegi.org.mx/geo/contenidos/topografia/compendio.aspx

[Último acceso: 10 Diciembra 2015]
López, B.L. (1991). Cultivos herbáceos. Vol. 1 Cereales. Ed. Ediciones Mundi Prensa. Madrid, España. 307-385.

Mendoza, E.M., Mosqueda V. C., Rangel L.J.A., López B. A., Rodríguez H.S.A., Latournerie M. L., Moreno M. E. (2006). Densidad de población y fertilización nitrogenada en clorofila, materia seca y rendimiento de maíz normas y QPM. Agricultura Técnica en México. 32(1):89-99

Molina, G.J.D. (1992). Introducción a la genética de poblaciones y cuantitativa: algunas implicaciones en genotecnia. Ed. AGT. México D. F. 349 p.

Palomo, G. R. (1976). La interacción genotipo-ambiente en el cultivo del algodonero en la comarca Lagunera. In: Memoria del VI Congreso Nacional de la SOMEFI, Monterrey, N. L. 348-352.

Reyes, C. P. (1990). El maíz y su cultivo. Ed. AGT. Editor, S.A. Primera Edición. México, D. F. 66-68.

SARH-INIFAP-PROCATI. (1991). Marco de referencia y Catalogo de Tecnologías Agropecuarias para el Distrito de Desarrollo Rural 004 Celaya. Campo Experimental Bajío. Publicación Especial No. 29. 210.

SIAP. Servicio de Información Agroalimentaria y Pesquera. (2017). http://www.siap.gob.mx/cierre-de-la-produccion-agricola-por-cultivo/ (Consulta 15 de junio, 2015).

Sinchi, P.J.H. (1991). Prueba de adaptación de 24 genotipos de maíz (Zea mays L). Campo experimental de Apodaca, N. L. en la primavera de 1990. Tesis de Maestría. ITESM. Campus Monterrey, México. 68 pp.

USDA. United States Department of AgricultureForeign Agricultural Service. (2015). Producción mundial de granos y cereales. http://www.usda. gov/wps/portal/usda/usdahome (Consulta 8 de julio, 2015).

Wong, R.R., Gutiérrez R.E., Palomo G.A., Rodríguez H.S.A., Córdoba O.H., Espinoza B.A., Lozano G.J.J. (2007). Aptitud combinatoria de componentes de rendimiento en líneas de maíz para grano en la comarca lagunera, México. Revista Fitotecnia Mexicana. 30(2):181-189. 


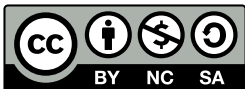

Licencia de Creative Commons

Revista de Investigación Agraria y Ambiental is licensed under a Creative Commons Reconocimiento-NoComercial-CompartirIgual 4.0 Internacional License. 
